\title{
WELL DETERMINED ATMOSPHERIC PARAMETERS FROM HIGH S/N RETICON SPECTRA OF FOUR G AND K DWARFS WITHIN 10 pc OF THE SUN
}

\author{
M.-N. Perrin ${ }^{1}$, G. Cayrel de Strobel ${ }^{2}$ and M. Dennefeld ${ }^{3}$ \\ ${ }^{1}$ Observatoire de Paris, Paris, France \\ ${ }^{2}$ Observatoire de Paris, Section de Meudon, Meudon, France \\ ${ }^{3}$ Institut d'Astrophysique, Paris, France
}

\begin{abstract}
The detailed analysis of high resolution, high signal-to-noise spectra of four $\mathrm{G}$ and $\mathrm{K}$ dwarfs lying within $10 \mathrm{pc}$ of the Sun is presented. This analysis is part of a project aiming at building up a homogeneous set of reliable atmospheric parameters for all $\mathrm{F}, \mathrm{G}$ and $\mathrm{K}$ stars nearer than $10 \mathrm{pc}$ according to the Gliese catalogue.
\end{abstract}

High resolution $(\Delta \lambda \simeq 0.13 \AA)$, high signal-to-noise $(\mathrm{S} / \mathrm{N}>200)$ spectra in four spectral intervals centered at $\lambda 6165,6560,6715$ and $8520 \AA$ have been obtained for four $\mathrm{G}$ and $K$ dwarfs lying within 10 parsecs of the Sun, HD 115617, HD 125072, HD 156274 and HD 156384, using the coude Echelle Spectrograph (CES) fed by the $1.4 \mathrm{~m}$ coudé Auxiliary Telescope (CAT) at ESO. The stars were chosen at random among those of the 69 nondegenerate stars of the Gliese catalogue (1969) with $\mathrm{T}_{\text {eff }}>4000 \mathrm{~K}$ and $\pi>0 .{ }^{\prime \prime} 100$, which have not yet been analysed in detail. The following results were derived.

The detailed analysis of the spectra in the $\lambda 6165,6560$ and $6715 \AA$ intervals, based on atmospheric models of Gustafsson (Gustafsson et al. 1975, Gustafsson, 1981), has provided the well determined atmospheric parameters of the stars, given in Table I.

Table I. Relevant data for the program stars

\begin{tabular}{lcccc}
\hline & HD 115617 & HD 156274 & HD 125072 & HD 156384 \\
\hline Sp.T. & G6V & G8V & K3V & K3V \\
V (mag.) & 4.74 & 5.53 & 6.66 & 6.10 \\
$\pi^{\prime \prime}$ & 0.113 & 0.133 & 0.106 & 0.140 \\
$\mathrm{~T}_{\text {eff }}(\mathrm{K})$ & 5585 & 5295 & 4965 & 4930 \\
$\sigma_{\mathrm{T}_{\text {ef }}}(\mathrm{K})$ & \pm 30 & \pm 60 & \pm 60 & \pm 100 \\
$\log \mathrm{g}$ & 4.5 & 4.5 & 4.5 & 4.5 \\
$\sigma_{\log \mathrm{g}}$ & \pm 0.2 & \pm 0.2 & \pm 0.2 & \pm 0.2 \\
$\left.\xi_{\mathrm{t}}(\mathrm{kms})^{-1}\right)$ & 1.0 & 0.5 & 0.5 & 0.5 \\
{$[\mathrm{Fe} / \mathrm{H}]_{\odot}^{*}$} & -0.02 & -0.35 & +0.26 & -0.59 \\
$\left.\sigma_{[\mathrm{Fe} / \mathrm{H}}\right]_{\odot}^{*}$ & \pm 0.03 & \pm 0.07 & \pm 0.09 & \pm 0.08 \\
\hline
\end{tabular}

The range of metallicity obtained for a very small randomly selected sample is surprisingly large : $[\mathrm{Fe} / \mathrm{H}]_{\odot}^{*}$ from -0.59 to +0.26 dex. 
A check for chromospheric activity was provided by the analysis of the two lines of the Ca II infrared triplet, lying in the $\lambda 8520 \AA$ interval. For the three stars observed in this interval, HD 115617, HD 125072 and HD 156274, the depth of the two Ca II lines, as compared with the filled in lines of the very active solar-type Hyades dwarf VB 64 (Cayrel et al. 1983), is indicative of low chromospheric activity.

From the analysis of the lithium feature in the $\lambda 6715 \AA$ region, it is derived that no lithium feature is apparent in the four stars.

More detailed results will be published in Astronomy and Astrophysics.

\section{REFERENCES}

Cayrel,R., Cayrel de Strobel,G., Campbell,B. Mein,N., Mein,P., Dumont,S.: 1983, Astron. A strophys. 123, 89

Gliese,W.: 1969, Veröff. Astron. Recheninst. Heidelberg. 22

Gustafsson,B.: 1981 (private communication)

Gustafsson,B., Bell,R.A., Eriksson,K., Nordlund,A.: 1975, Astron. Astrophys. 42, 407 